\title{
A essência da infinitude do conjunto dos números primos
}

\author{
The infinity essence of all prime numbers
}

Luciana Maria Da Silva ${ }^{1}$ lumasilva15@gmail.com

Esdras Jafet Aristides da Silva ${ }^{2}$ esdras.jafet@upe.br

\begin{abstract}
Resumo
Neste trabalho abordaram-se questões relacionadas à infinitude do conjunto dos números primos, entendidas originalmente como a não existência do maior desses números, foi demonstrada por Euclides (provavelmente o primeiro) por volta do ano 300 antes de Cristo, quando buscava caracterizar os chamados números perfeitos. Entretanto, para a matemática moderna, ainda é possível classificar os conjuntos infinitos quanto ao seu tamanho: "infinitos grandes" ou "infinitos pequenos". Neste contexto, quão grande é o conjunto dos números primos? Na busca pela resposta a esta questão deparou-se com outras tantas demonstrações do teorema de Euclides, relacionando variadas áreas da matemática, que se buscou por bem revisitar a questão da infinitude dos números primos de maneira mais completa. Então, para responder estas questões, resgataram-se algumas dessas interessantes, importantes e engenhosas demonstrações.
\end{abstract}

Palavras-chave: Números primos; Euclides; Infinitude; Demonstrações.

\begin{abstract}
In this paper discussed the issues related to the infinity of all prime numbers, originally understood as the absence of these higher numbers, was demonstrated by Euclid (probably the first) about the year 300 b.C., when sought characterize the so-called perfect numbers. However, for the modern mathematics, it is still possible to classify the infinite sets about its size: "big infinity" or "small infinity". In this context, how the size is all the prime numbers? In the search for the answer to this question we are faced with so many demonstrations of the theorem of Euclid, linking different areas of mathematics, we believe in and review the question of infinity of prime numbers more completely. Then to answer these questions, recovering some of these interesting, important and ingenious demonstrations.
\end{abstract}

Keywords: Primes numbers; Euclid; Infinity; Demonstrations.

\section{Introdução}

A palavra "primo" se refere à ideia de primeiro, e teve sua origem numa velha concepção numérica dos pitagóricos. Provavelmente, a noção de número primo foi introduzida por Pitágoras, 530 a.C., sendo que a mesma representou um papel central tanto na matemática quanto no misticismo pitagórico (Dos Santos, 2003). Vale ressaltar que não se tem completa convicção quanto a esta atribuição, uma vez que, Pitágoras e seus seguidores não deixaram nenhum escrito. Os únicos documentos mais antigos que se tem falando de suas

\footnotetext{
${ }^{1}$ Licenciada em Matemática pela UPE, Mestre em Ciências Geodésicas e Tecnologia da Geoinformação pela UFPE, Doutoranda em Ciências Geodésicas pela UFPR, Curitiba-PR, Brasil. Rua Capitão João Ribas de Oliveira, 415, Ap-23 Bl-B, Bairro - Guabirotuba, CEP-81510-350, Curitiba-PR, Brasil. Email:lumasilva15@gmail.com.

2 Bacharel em Matemática pela UFPE, Mestre em Matemática pela UFPE, Doutorando em Educação Matemática pela UFPE. Professor na Universidade de Pernambuco - Campus Mata Norte (UPE/CMN), Departarmento de Matemática. E-mail: esdras.jafet@upe.br.
} 
ideias se resumem a pequenos fragmentos de textos escritos de várias gerações depois deles. Nestes fragmentos continham informações escassas, mas são unânimes em ratificar que o estudo dos números primos foi iniciado por Pitágoras (Da Silva e Da Silva, 2009).

O livro de matemática mais antigo que chegou completo aos nossos tempos e que desenvolve sistematicamente o estudo dos números primos é “Os Elementos” escrito por Euclides por volta de 300 a.C, traduzido por Bicudo (2009). Ele seguiu muito de perto as orientações matemáticas dos pitagóricos. No capítulo em que trata da Teoria dos Números, ele define número primo de um modo absolutamente compatível com as ideias pitagóricas.

Os Gregos foram os primeiros a estudarem intensivamente os números primos e suas propriedades. Dentre os vários resultados obtidos, eles mostraram que esses números se encontram espaçados de forma irregular, o que impedia de uma "fórmula capaz de produzir primos". Duas questões foram básicas nesta época: Quantos são os números primos? e Como saber se um dado número é ou não primo?

A infinitude do conjunto dos números primos é a essência dos números inteiros, entendida originalmente como a não existência do maior desses números. Sendo que, o conceito de infinitude gerava muita controvérsia, surgindo muitas perguntas em relação ao conceito de infinitude e ao conjunto de primos, fazendo que chegasse a refletir: Como pode alguma coisa que é inegavelmente menor do que uma quantidade infinita ser infinita?

Singh (2008, p. 108) destaca que no começo do século XX o matemático alemão David Hilbert enfatiza a questão do infinito, com a seguinte sentença: O infinito! Nenhum outro conceito estimulou profundamente o espírito humano; nenhuma outra ideia estimulou o intelecto de modo tão frutífero, e, no entanto nenhum outro conceito precisa ser mais esclarecido do que a ideia de infinito.

No livro VII de Os Elementos, Euclides provou uma versão preliminar do Teorema de Fatoração Única (TFU), a qual estabelece que: "Todo número composto é divisível por algum número primo”. A partir deste resultado foi possível evidenciar a importância dos números primos, reconhecida até hoje. Segundo Singh (2008, p. 130), os teóricos dos números consideram os números primos como os mais importantes entre todos os números porque eles são como os átomos da matemática. Os números primos são os tijolos da construção numérica porque todos os outros números podem ser criados multiplicando-se combinações de números primos.

Com base na versão preliminar do TFU que Euclides apresentou, na proposição 20 do livro IX, o que parece ser a primeira demonstração de que o conjunto dos números primos é infinito. Para a matemática moderna, ainda é possível classifica-los quanto ao seu tamanho. 
Neste contexto em relação ao tamanho do conjunto, busca-se por uma resposta. O Teorema de Euclides está relacionado com outras demonstrações da infinitude dos números primos, relacionando distintas áreas da matemática. Diante dos argumentos apresentados, abordaramse as questões em relacionadas a distintas áreas da matemática, resgatando três variantes demonstrações de Euclides, adicionalmente as demonstrações atribuídas aos matemáticos Polya, Schorn e Euler, utilizando como referência principal Ribenboin (1989), de maneira didática e autocontida.

\section{Demonstração de Euclides}

Euclides estudando os chamados números perfeitos que são os que seus divisores produzem o dobro deles mesmos, estudados por Pitágoras, ele não se contentou em apenas colecionar tais números; buscava dar um significado mais profundo a eles. Ele observou que à medida que os números inteiros se tornam maiores, a tarefa de encontrar números perfeitos se tornava mais difícil e que esta também é uma característica dos números primos. Em suas pesquisas ele estabeleceu que a totalidade dos números primos fosse maior que qualquer quantidade pré-fixada.

Baseado em uma versão preliminar do Teorema Fundamental da Aritmética (TFA), usou a técnica da demonstração por absurdo (método indireto), apresentando uma demonstração elegante e simples da infinitude dos números primos, mais detalhes em Pimentel (2006). Utilizando o TFA e a demonstração por absurdo, Euclides admitiu como hipótese que a quantidade de números primos positivos é finita, considerando três números primos. A partir disto mostrou que deveria haver outro primo diferente dos primos préfixados, pois se assim não o fosse, uma contradição à definição de número primo seria obtida.

$\mathrm{Na}$ versão original do Teorema de Euclides, ele dizia que um número era mensurado por outro quando ele era divisível por este, enquanto que os fatores de um número eram chamados de parte deste número, chegando à definição do número primo no Capítulo VII: Um número é primo quando é mensurado pela unidade. Esta definição é diferente da que se tem usual, pois número é diferente de unidade. A terminologia usual tem-se que: "Um número natural p é primo se for diferente de 1 e se possui exatamente dois divisores: 1 e o próprio p.

A partir da definição de número primo, definiu os números que não eram primos de compostos: Um número é composto se for mensurado por algum número. Com a visão da TFU chegou à conclusão que: Qualquer número composto é mensurado por algum número primo. A partir desta definição, utilizando os resultados como base para a sua demonstração da infinitude dos primos. 
Na proposição 20 do livro IX de Os Elementos, enunciou considerando que se $A, B e$ C são números primos, haveria mais primos além destes, essa demonstração foi apresentada da seguinte forma: Pegue ED o menor número mensurado por $A, B$ e $C$ simultaneamente. Adicione a unidade DF a ED obtendo o número EF. Então ou EF é primo ou é composto. Se EF é primo, então acabou, pois EF é maior que $A, B$ e $C$; senão, de acordo com a versão preliminar do TFA, EF é mensurado por um primo que será denotado por G. Eu afirmo que $G$ é diferente de $A, B$ e $C$. De fato, se isto não fosse verdade então ED é mensurado por $G$ o que implica que o resto da divisão de EF por ED é mensurado por G. Mas, o resto é a unidade e, portanto $G$ é a unidade, o que gera uma contradição com a definição de número primo".

Hefez (2005) em contexto mais algébrico apresenta a versão contemporânea de divisibilidade: Um número inteiro $b \neq 0$ divide o inteiro se e somente se existe um outro inteiro c tal que $a=b^{*}$ c. Neste caso, escreve-se que $b \mid a$ para indicar que $b$ divide $a$.

$\mathrm{Na}$ literatura atual encontra-se a seguinte demonstração que representa uma pequena generalização da demonstração de Euclides: Suponhamos que existem apenas $r$ números primos positivos, digamos $p_{1}, p_{2}, p_{3}, \ldots, p_{r}$, para algum $r \in N$ pré-fixado (de fato, na demonstração de Euclides tem-se $r=3$ ). O número natural $n=p_{1} p_{2} \ldots p_{r}+1$ (o maior número mensurado por esses primos acrescido da unidade) ou é primo ou é composto. Se né primo, então acabou uma vez que $n>p_{i}$ para cada $i \epsilon\{1, \ldots, r\}$, ou seja, um novo primo foi encontrado. Se né composto, então pela versão preliminar do TFU, existe um primo p tal que $p \mid n$. Se $p=p_{i}$ para algum $i$, segue do algoritmo de divisão que $p \mid\left(n-p_{1} p_{2} \ldots p_{r}\right)=1$, disto $p=1$ que é uma contradição. Portanto p é primo diferente daqueles listados, e obtemos assim um novo número primo.

\subsection{Algumas variantes da demonstração de Euclides}

As demonstrações atribuídas aos matemáticos: Ernest Kummer, Thomas J. Stieljes e Charles Hermite, assim como a demonstração de Euclides são simples e elegantes. Utilizouse Ribenboin (1991) para a análise dessas demonstrações.

Kummer ao estudar os números primos chamou a atenção para o fato de que a TFU diz que só existe uma combinação de números primos que, ao serem multiplicados, produzirão determinados números, adicionalmente ao fato de que não existia na matemática algo que pudesse abordar todos os primos irregulares de uma única vez. Ele enfocou que a partir do estudo das técnicas elaboradas para cada primo irregular, cada caso poderia ser resolvido individualmente, mas o desenvolvimento seria muito lento e penoso. Devido que os 
números primos irregulares eram infinitos e para solucionar este problema individualmente seria necessário ocupar todos os matemáticos do mundo pelo resto da eternidade (SINGH, 2008, p. 127).

De acordo com Ribenboim (1991), em 1958 Kummer apresentou a seguinte demonstração: Suponhamos que existem apenas $p_{1}, p_{2}, p_{3}, \ldots, p_{r}$ primos positivos, onde $r$ é algum número natural pré-fixado. Uma vez que o conjunto dos números naturais é infinito, precisa existir um natural $n>2$ tal que $n$ e $n-1$ são divisiveis por um mesmo primo $p$ deste conjunto. De fato basta considerar $n=p_{1} p_{2} \ldots p_{r}$. Assim como Euclides, Kummer chega a uma contradição, isto implica que $p \mid n-(n-1)=1$. Sendo assim, Kummer afirma que o conjunto dos números primos é infinito baseado na contradição e no TFU.

Stieltjes apresentou sua demonstração baseada na seguinte caracterização equivalente de número primo: Um natural $p>1$ é primo se e somente se, se dados $a, b \in Z$, p ab implica $p \mid a$ ou $p \mid b$ (HEFEZ, 2005, p.82).

Segundo Ribenboim (1991), em sua demonstração Stieltjes escreveu: "Suponhamos que existem apenas $p_{1}, p_{2}, p_{3}, \ldots, p_{r}$ primos positivos, onde $r$ é algum número natural préfixado. Seja $N=p_{1} p_{2} \ldots p_{r}$. Desde que cada $p_{i}$ divide $N$, o natural $N$ não pode ser primo. De acordo com o TFA podemos fatorar $N$, isto é, podemos escrever $N=m n$ com $1<m, n<N$. Pela caracterização de número primo dada acima, e pelo fato dos primos $p_{1}, p_{2}, p_{3}, \ldots, p_{r}$ serem distintos, para cada $i$, o primo $p_{i}$ divide $m$ ou $n$ mas nunca divide os dois ao mesmo tempo. Isto implica que nenhum dos primos $p_{i}$ pode dividir o número natural $m+n$ donde segue que $m+n=1$, contradizendo as hipóteses sobre $m$ e $n "$.

A demonstração de Hermite, talvez seja a demonstração mais bela de todas as demonstrações da Teoria de Euclides, por sua simplicidade. Assim como Kummer e Stieltjes, ele usou o TFA, mas a ideia central era mostrar que dado um natural $n$, sempre existe um número primo $p$ maior que $n$. Com isto, ele conseguiu realizar uma demonstração direta do Teorema de Euclides, pois a infinitude dos números naturais implica na infinitude dos primos. Sua demonstração é: "Dado um número natural $n$ considere $N=n !+1$, onde $n !=1 * 2 \ldots n$ corresponde ao fatorial de $n$. Pelo TFA, existe um primo $p$ dividindo $N$, onde é necessário notar que um tal primo $p$ não pode ser menor que $n$. De fato, se assim o fosse, ele necessariamente apareceria na expressão $n !=1 * 2 \ldots p \ldots(n-1) * n$, o que implica na contradição $p=1$, pois $p \mid N-n !=(n !+1)-n !=1 ”$. 


\section{Demonstrações do Teorema de Euclides conectadas a outras Teorias Matemáticas}

O Teorema de Euclides serviu como base para outras demonstrações oriundas de teorias matemáticas que, em princípio, não estão diretamente relacionadas com os números primos. Embora mais sofisticadas e algumas vezes muito engenhosas, a beleza destas demonstrações está no fato de exemplificar como variadas áreas da matemática, aparentemente sem relação umas com as outras podem revelar algum tipo de conexão.

\subsection{Demonstração de Polya e os Números de Fermat}

Primeiramente destaca-se a demonstração de George Polya. Ele fundamenta-se no TFA não seguindo os mesmos moldes da demonstração de Euclides, pois ele utiliza os Números de Fermat para mostrar que quaisquer dois deles são primos entre si. Ou seja, ele se baseia no conceito, bastante simples, de números primos entre si ou números primos relativos: “Dois números são ditos primos entre si se o máximo divisor comum entre eles é um”.

Segundo Hefez (2005), dados dois inteiros $a$ e $b$, então um máximo divisor comum de $a$ e $b$ é um inteiro $d$, quando satisfaz as seguintes propriedades:

1. $d \mid a$ e $d \mid b$;

2. Se $c$ é um inteiro satisfazendo (1), então $c \mid d$.

Nota-se que $d$ é um máximo divisor comum de $a$ e $b$ então $-d$ também é. O máximo divisor comum positivo de $a$ e $b$ será denotado por $m d c(a, b)$.

Dessa forma, se $a$ e $b$ são primos entre si, não existe um primo $p$ que divide $a$ e $b$ simultaneamente. Daí, se $1<a 1<a 2<\ldots<a n<\ldots$ é uma sequência infinita de números naturais dois a dois primos entre si. E se $p_{i}$ é um primo dividindo $a_{i}$, que existe pelo TFA, tem-se sempre $p_{i} \neq p_{j}$ se $i \neq j$. Portanto a sequência dos distintos números primos $\left\{p_{i}\right\}$ é uma sequência infinita.

A sequência escolhida por Polya era formada de números muitos conhecidos à época, os chamados Primos de Fermat, que de acordo com Hefez (2005, p. 97), eram definidos da seguinte maneira: "Seja $n>0$ um número natural. $O$ n-ésimo primo de Fermat é o número da forma $F_{n}=2^{2^{n}}+1$.

É fácil notar que, se os Primos de Fermat fossem realmente primos, como Fermat acreditava, teríamos uma demonstração do Teorema de Euclides. Ou seja, teríamos algo muito mais importante, uma fórmula para gerar números primos, algo procurado e até hoje não encontrado. Euler, em 1732, que mostrou que nem todos os primos de Fermat são primos, mais detalhes em Coutinho (2005, p.58). 
Para demonstrar utilizando os números de Fermat, Polya seguiu a ideia de que não se precisava que os Primos de Fermat fossem primos, bastava que eles fossem primos entre si. Utilizando o Princípio de Indução Finita (PIF) ou Princípio de Indução Matemática (PIM), pode-se demonstrar a ideia de Polya mais detalhes em (HEFEZ, 2005, p. 8; LIMA, 2006a, p. 33). Sendo PIF ou PIM uma das técnicas matemáticas mais utilizadas em demonstrações.

Sendo assim o PIM ou PIF aborda os seguintes aspectos: Seja a $\in N$ e seja $p(n)$ uma sentença aberta em n. Suponha que:

(i) $\quad p(a)$ é verdade, e que

(ii) $\quad \forall n \geq a, p(n) \Rightarrow p(n+1)$ é verdade, então, $p(n)$ é verdade para todo $n \geq a$.

A demonstração de Polya é apresentada utilizando os princípios básicos dos números de Fermat, como mencionado anteriormente, então de acordo com Ribenboim (1991), ela pode ser escrita da seguinte forma:

É suficiente encontrar uma sucessão natural de números $1<a 1<a 2<\ldots$, que sejam primos relativos dois a dois, isto é sem fatores primos em comum. Logo, se p pé um primo que divide $a_{1}$, se, $p_{2}$ é um primo que divide $a_{2}$. E assim sucessivamente, então $p_{1}, p_{2}, p_{3}, \ldots$, são todos diferentes. Nesta demonstração os números $a_{n}$ serão escolhidos como sendo Números de Fermat, onde: $F_{n}=2^{2^{n}}+1(n \geq 0)$, ou simplesmente $F_{m}=4^{m}+1$. De fato é fácil de ver por indução em $m$, então: $F_{m}=4^{m}+1$, pode ser fatorado como $F_{m}=\left(2^{m}+1\right)\left(2^{m}-1\right)+$ 2, logo teremos: $F_{m}-2=\left(2^{m}+1\right)\left(2^{m}-1\right)$. Então $F_{m}-2$ pode ser escrito como a igualdade de uma sequência infinita de números naturais, onde: $F_{m}-2=F_{0} F_{1} \ldots F_{m-1}$. Consequentemente, se $n<m$, então, $F_{n}$ divide $F_{m}-2$. Se um número primo $p$ dividisse $F_{n}$ e $F_{m}$ então dividiria $F_{m}-2$ e $F_{m}$, por isso também dividiria 2, logo $p=2$. Mas $F_{n}$ é ímpar, consequentemente não é divisível por 2. Isto mostra que os Números de Fermat são primos relativos dois a dois.

\subsection{Demonstração de Schorn e o Axioma de Boa Ordem}

A demonstração de Schorn é considerada bastante simples, mas se fundamenta em resultados considerados com precisão da matemática que demorou muito tempo para ser aceito. Vale ressaltar que o PIM se fundamenta no Axioma de Boa Ordem, que é equivalente ao Axioma da Escolha e o lema de Zorn, mas que os resultados ainda hoje não são aceitos, mais detalhes em (HENLE, 1996; COELHO e MILIES, 2006).

Schorn realizou sua demonstração sem usar diretamente indução, embora seja uma variante da demonstração de Polya, ele produz uma sequência infinita de números positivos entre si. A ideia da demonstração é que a construção da sequência de números primos entre si 
apresentada por Schorn se baseia no fato de que se $1<i<j<n$, então $\operatorname{mdc}(n ! i+1, n ! j+1)=1$. De fato, escrevendo $j=i+d$ quando $1 \leq d<n$, se e somente se $\operatorname{mdc}((n !) i+1,(n !) j+1)=\operatorname{mdc}((n !) i+1$, $(n !) d)=1$ mas todo primo $\mathrm{p}$ dividindo $(n !) d$ ou é igual a $n$.

Para realizar a demonstração assume-se que existe somente m números primos, se $n=m+1$. O procedimento da ideia diz que se $n$ inteiro (n!) $i+1$ (para $i=1,2,3, \ldots, n$ ) são relativamente primos ímpares. Se $p_{i}$ é um primo dividindo $(n !) i+1$, quando $p_{1}, p_{2} \ldots p_{m}$ são todos primos distintos, com $n=m+1$, isto é absurdo.

\subsection{Demonstração de Euler e as Séries Numéricas}

Para realizar sua demonstração Euler utiliza o TFA, mas não seguiu a linha de demonstração de Euclides. Quando Euler supõe que existe uma quantidade finita de primos, ele obteve uma contradição a partir de expressões envolvendo somas infinitas, as chamadas séries numéricas, só entendidas através de limites. Assim sendo, é de se esperar que algumas somas possam ser efetuadas (convirjam) e outras não, já que nem toda sequência possui limites (LIMA, 2006b, p. 134).

A ideia da demonstração de Euler é que dada uma lista de números reais $a_{1}, a_{2}, \ldots, a_{n}, \ldots$ é chamada de sequência (infinita) de números reais e é usualmente denotada por $\left(a_{n}\right)_{n=1}^{\infty}$. Os números $a_{i}$ são chamados de termos da sequência, e soma de todos estes termos é chamada de série de números reais associada à sequência. Usamos $\sum_{n=1}^{\infty} a_{n}$ para representar esta série, isto é: $\sum_{n=1}^{\infty} a_{n}=a_{1}+a_{2}+\ldots+a_{n}+\ldots$. Do lado direito da igualdade existem infinitas parcelas, é preciso atribuir um significado a este somatório infinito. Este tipo de situação não é de todo desconhecido. Recordemos que dados dois números reais $a$ e $r$ a sequência definida por $a_{1}=a$ e $a_{n}=a_{n-1} * r$ se $n>1$ é chamada de Progressão Geométrica $(P G)$ de primeiro termo igual a a e razão $r$. A série associada a uma sequência deste tipo é chamada de Série Geométrica. Neste caso particular, o termo $a_{n}$, usualmente chamado de Termo Geral tem a forma $a_{n}=a * r^{n-1}$, o que permite escrever: $\sum_{n=1}^{\infty} a * r^{n-1}$. É relativamente fácil mostrar que esta série pode ser associada a um número real se e somente se $|a|<1$. Neste caso, este número real corresponde a $\frac{a}{1-r}$ e escrevemos $\sum_{n=1}^{\infty} a * r^{n-1}=\frac{a}{1-r}$. Quando é possível associar a uma série um número real, dizemos que a série é Convergente, caso contrário a série será Divergente. O exemplo clássico de série divergente é a chamada série harmônica dada por $\sum_{n=1}^{\infty} \frac{1}{n}$. Uma demonstração de que esta série de fato diverge pode ser encontrada como, por exemplo, em Ávila (2006, p. 109). 
Euler mostrou que, na hipótese de existir apenas um número finito de primos, digamos $p_{1}, p_{2}, p_{3}, \ldots, p_{n}$ é possível obter uma igualdade em que de um lado está uma série divergente e do outro um número real, o que é, segundo a definição acima, um absurdo.

A observação crucial de Euler foi a seguinte. Se $p$ é um número primo, então $\frac{1}{p}<1$, o que implica em $\sum_{n=0}^{\infty}\left(\frac{1}{p^{k}}\right)=\sum_{n=0}^{\infty}\left(\frac{1}{p}\right)^{k}=\frac{1}{1-\frac{1}{p}}$. Então, se $q$ é outro primo obtemos: $\sum_{n=0}^{\infty}\left(\frac{1}{p^{k}}\right) * \sum_{n=0}^{\infty}\left(\frac{1}{q^{k}}\right)=\left(\frac{1}{1-\frac{1}{p}}\right) *\left(\frac{1}{1-\frac{1}{q}}\right)$. Após verificarmos as propriedades operacionais usuais também são válidas para as séries convergentes, o lado esquerdo da igualdade corresponde: $1+\frac{1}{p}+\frac{1}{p^{2}}+\frac{1}{p q}+\frac{1}{q^{2}}+\cdots$. Esta expressão corresponde ao somatório de todas as frações do tipo $\frac{1}{p^{k} q^{h}}$, com $\mathrm{k}, \mathrm{h} \geq 0$, isto é, corresponde a somar todas as parcelas do tipo $\frac{1}{n}$, onde $n$ é um natural cuja fatoração é o produto de potências de primos $p$ e $q$.

Então para a Euler deve existir infinitos números primos pelo fato de uma certa expressão formada por todos os números primos ser infinita. Supondo que $p_{1}, p_{2}, p_{3}, \ldots, p_{n}$ são todos números primos. Para cada $\mathrm{m}=1, \ldots, \mathrm{n}$, então $\sum_{k=0}^{\infty}\left(\frac{1}{p_{m}^{k}}\right)=\left(\frac{1}{1-\frac{1}{p_{m}}}\right)$. Multiplicando estas n igualdades, obtém-se: $\prod_{m=1}^{n} \sum_{k=0}^{\infty}\left(\frac{1}{p_{m}^{k}}\right)=\prod_{m=1}^{n}\left(\frac{1}{1-\frac{1}{p_{m}}}\right)$, onde $1 \leq \mathrm{m} \leq \mathrm{n}$ e $\sum_{k=0}^{\infty}\left(\frac{1}{p_{m}^{k}}\right)=\left(\frac{1}{1-\frac{1}{p_{m}}}\right)$ é a soma dos inversos de todos os naturais, cada um contando apenas uma vez, isto segue o teorema que diz que cada número natural é igual, de um só modo, a produto de números primos. Mas a série $\sum_{n=1}^{\infty}\left(\frac{1}{n}\right)=\prod_{p}\left(\frac{1}{1-\frac{1}{p^{r}}}\right)$ para $(\mathrm{r}>1)$. Então a soma do inverso de números primos é divergente $\sum_{p}\left(\frac{1}{p}\right)=\infty$ por ser uma série de termos positivos, a ordem de sua soma é irrelevante, logo a parte esquerda é infinita enquanto que a parte direita é claramente finita. Isto é um absurdo, pois o limite das séries deveria claramente ser igual.

\section{Discussões da Visão Clássica à atual dos Números Primos}

A história dos números primos é interessante devido relatar o início do descobrimento dos números primos, que teve início na escola pitagórica tendo como “professor” Pitágoras, sendo que pouco se sabe em relação a ele, pois ele nada deixou escrito. Os gregos foram os primeiros a perceber que os números primos são infinitos e que todo 
número inteiro podia ser gerado pela multiplicação destes números. Com o passar dos tempos Euclides interessa-se em dar continuidade ao que Pitágoras "começou estudar" escrevendo a demonstração que provava a infinitude dos primos.

A partir deste fato, surgem novas demonstrações escritas por outros matemáticos provando a infinitude dos primos, alguns seguiram o mesmo molde da demonstração de Euclides, outros escreveram usando as mesmas ideias, mas de forma mais engenhosa. Quanto aos fatos em um encadeamento dedutivo em que um reduzido número de proposições e definições iniciais são utilizadas para demonstrar, um após outros, todo os teoremas considerados podem ser vistos inicialmente de forma organizada no livro Elementos de Euclides.

Os elementos de Euclides (traduzido por Bicudo, 2009) são praticamente tudo o que se tem da matemática grega que se desenvolveu desde seu início com Tales de Mileto, até o tempo de Euclides. Esse é um período de cerca de 250 anos, muito pouco tempo para que a matemática, logicamente organizada, evoluísse do estágio embrionário em que se encontrava com Tales, até o alto grau de sofisticação que transparece nos Elementos (AVILA, 2006, p. 19).

Há muitos anos os números primos vêm servindo de estudos devido sua importância e o mistério que "escondem" em seu espaçamento de forma irregular, pois não se sabe a razão que eles seguem. Os números primos desempenham um papel importante em vários ramos da Matemática, como: Álgebra, Geometria, Análise, Teoria dos Números, Topologia e a Criptografia, como apresentado nas distintas demonstrações neste trabalho.

Durante muito tempo, o estudo dos primos foi "encarado" como um jogo intelectual, sem nenhuma importância para ser utilizado. Na criptografia, atualmente eles estão ocupando um espaço muito importante devido à transmissão de segurança que eles emitem: no sistema de codificação e decodificação de mensagens, na utilização de senhas bancárias, entre outras situações.

Estudar os números primos é uma forma de “desvendar" muitos conteúdos matemáticos e assim mostrar que a partir de apenas um assunto existem muitos outros entrelaçados, além de modificar a visão errônea que muitas pessoas têm em relação aos números primos, pois muitas acham que apenas se necessita saber defini-lo.

O mundo globalizado exige cada vez mais meios seguros de proteger senhas secretas que anteriormente eram facilmente descobertas. Os números grandes quão grandes são possibilitam essa proteção. Basta serem bem escolhidos para que as senhas estejam protegidas e dificulte o descobrimento destas, pois, Uma grande descoberta envolve a solução de um 
grande problema, mas há uma semente de descoberta na solução de qualquer problema. Seu problema pode ser modesto; porém, se ele desafiar sua curiosidade e fizer funcionar sua capacidade inventiva, e caso você o resolva sozinho, então você poderá experimentar a tensão e o prazer do triunfo da descoberta. George Polya (STEWART, 2006, p. vii).

Adicionalmente, vale ressaltar que, ferramentas matemáticas têm facilitado a obter um número primo grande em um determinado intervalo dentre elas pode-se destacar softwares ou linguagens de programação como: Python, Maple, Matlab, entre outras.

\section{Conclusões}

Esta pesquisa foi desenvolvida tendo como base a história dos números primos e incrementar as demonstrações realizadas por muitos matemáticos que o conjunto dos números primos é infinito. Além disso, mostrar os diferentes caminhos que a matemática evoluiu, não estando ligados diretamente a uma problemática especifica, buscando e entrelaçando vários assuntos distintos. Demonstrar não é tão simples, todas as demonstrações necessitam de um embasamento teórico e de muita cognitividade.

A vida dos matemáticos é "impulsionada" por uma vontade de resolver os problemas que encontravam ao longo do tempo. Muitas demonstrações para provar a infinitude dos primos surgem no intuito de provar que os conjuntos dos números primos são infinitos, utilizando uma contradição.

Euclides foi o marco inicial, ele utilizou ideias do Teorema Fundamental da Aritmética e prova ao absurdo, dando possibilidades para que surgisse uma versão contemporânea de sua demonstração, além de algumas variantes de sua demonstração. Merecendo destaque: Hermite, Kummer e Stieljes, eles utilizarem a mesma ideia para demonstrar a infinitude dos primos. Sendo que destes a demonstração de Hermite pode-se dizer que foi a mais simples e bonita. Outras demonstrações importantes que mereceram grandes destaques, pois muitos "matemáticos", de forma distinta e engenhosa organizaram suas ideias para desenvolver o teorema de Euclides e assim chegam ao esperado, uma demonstração que provasse a infinitude dos primos e utilizando conceitos matemáticos bastante dispare e um grau de dificuldade maior.

Euler utilizou ramos da matemática que envolve muitos conceitos e que é necessário salientar que estes, muitas vezes não são vistos nem no ensino superior. Sua demonstração é de forma muito engenhosa. Outros matemáticos ousaram em desenvolver sua demonstração mais fazendo um paralelo em relação à de Euler foram de forma mais simples, como a de 
Polya que utilizou os Números de Fermat como base fundamental e Schorn que escreveu sua demonstração inspirada em Polya.

A partir desta pesquisa observou-se que se pode trabalhar com muitos outros ramos da matemática integrada ao conceito de números primos, pois contribui para o desenvolvimento cognitivo. Vale ressaltar que a integração de primos com distintos ramos é possível realizar com alunos de ensino superior, o qual suscita um conhecimento mais aperfeiçoado e desenvolvido.

\section{REFERÊNCIAS}

AVILA, G. Análise Matemática para Licenciatura. São Paulo: Edgard Blucher, 2006

BICUDO, I. Os elementos: Euclides. São Paulo: UNESP, 2009.

COELHO, S. P.; MILIES, F. C. P. Números: Uma Introdução à Matemática. Editora: EDUSP, $3^{\text {a }}$ ed., 2006.

COUTINHO, S. C. Números inteiros e Criptografia RSA. São Paulo: IMPA, 2005.

DA SILVA, L. M.; DA SILVA, E. J. A. Números Primos: um conjunto infinito. Universidade de Pernambuco: Trabalho de Conclusão de Curso, 2009, 41p.

DOS SANTOS, M. F. Pitágoras e o Tema do Número. São Paulo: IBRASA, 2000.

HEFEZ, A. Elementos de Aritmética. Rio de Janeiro: SBM, 2005.

HENLE, J. M. An Outline of Set Theory. New York: Springer - Verlag, 1996.

LIMA, E. L. A Matemática do Ensino Médio. Rio de Janeiro: SBM, 2006a.

LIMA, E. L. Curso de Análise. Rio de Janeiro: SBM, 12. Ed, v.1, $2006 b$.

PIMENTEL, E. G. Teoria dos Números e Criptografia RSA. Universidade Federal de Minas Gerais, 2006,49 p.

RIBENBOIN, P. The New Book of Prime Number Record. Canadá: Springer, 1989.

RIBENBOIN, P. The Little Book of Prime. New York: Springer - Verlag, 1991

SINGH, S. O Último Teorema de Fermat. Rio de Janeiro: Record, 2008.

STEWART, J. Cálculo. São Paulo: Thomson Learning, 2006. 